\title{
Positive Periodic Solutions of a Neutral Impulsive Predator-Prey Model
}

\author{
Guirong Liu and Xiaojuan Song \\ School of Mathematical Sciences, Shanxi University, Taiyuan, Shanxi 030006, China \\ Correspondence should be addressed to Xiaojuan Song; 963940826@qq.com
}

Received 24 September 2014; Revised 23 January 2015; Accepted 24 January 2015

Academic Editor: Bai-Lian Larry Li

Copyright (C) 2015 G. Liu and X. Song. This is an open access article distributed under the Creative Commons Attribution License, which permits unrestricted use, distribution, and reproduction in any medium, provided the original work is properly cited.

\begin{abstract}
We investigate a ratio-dependent predator-prey model with Holling type III functional response based on system of neutral impulsive differential equations. Sufficient conditions for existence of positive periodic solutions are obtained by applying continuation theorem. Our main results demonstrate that under the suitable periodic impulse perturbations, the neutral impulsive system preserves the periodicity of the corresponding neutral system without impulse. In addition, our results can be applied to the corresponding system without impulsive effect, and thus, extend previous results.
\end{abstract}

\section{Introduction}

During the recent three decades, impulsive differential equations were intensively investigated. Some researchers devote themselves to the research on the theory of impulsive differential equations. The detailed introductions of impulsive differential equations were given in [1].

In the real world, there are many man-made and natural factors that always lead to sudden changes of population number at certain moments of time. For example, the births of many species are not continuous but happen at some regular time. These changes can often be characterized mathematically in the form of impulses. If these impulsive factors are incorporated into population dynamical models, the corresponding models should be governed by impulsive differential equations. With the development of impulsive differential equations, many models of impulsive differential equations have been considered extensively. For example, many authors have studied some qualitative properties of the solutions for impulsive differential equations such as periodicity [2-6], stability [7-10], and permanence and extinction $[2,11]$.

Recently, Du and Feng [12] studied the existence of periodic solutions for the following neutral impulsive predator-prey model with Beddington-DeAngelis functional response:

$$
\begin{gathered}
x^{\prime}(t)=x(t)\left[r(t)-a(t) x\left(t-\sigma_{1}(t)\right)-\rho(t) x^{\prime}\left(t-\sigma_{2}(t)\right)\right. \\
\left.-\frac{b(t) y(t)}{f(t)+n(t) x(t)+m(t) y(t)}\right], \\
y^{\prime}(t) \quad y(t)[-d(t) \quad \\
\left.+\frac{c(t) x\left(t-\sigma_{3}(t)\right)}{f(t)+n(t) x\left(t-\sigma_{3}(t)\right)+m(t) y\left(t-\sigma_{3}(t)\right)}\right], \\
\quad t \neq t_{k}, \quad k=1,2, \ldots, \\
x\left(t_{k}^{+}\right)-x\left(t_{k}\right)=\theta_{1 k} x\left(t_{k}\right), \\
y\left(t_{k}^{+}\right)-y\left(t_{k}\right)=\theta_{2 k} y\left(t_{k}\right), \\
t=t_{k}, \quad k=1,2, \ldots
\end{gathered}
$$


In 2011, Liu and Yan [13] studied the existence of periodic solutions for the following neutral ratio-dependent predatorprey model with Holling type III functional response:

$$
\begin{gathered}
x^{\prime}(t)=x(t)\left[r(t)-a(t) x\left(t-\sigma_{1}\right)-\rho x^{\prime}\left(t-\sigma_{2}\right)\right] \\
-\frac{c(t) x^{2}(t)}{m^{2} y^{2}(t)+x^{2}(t)} y(t), \\
y^{\prime}(t)=y(t)\left[-d(t)+\frac{h(t) x^{2}(t-\tau(t))}{m^{2} y^{2}(t-\tau(t))+x^{2}(t-\tau(t))}\right] .
\end{gathered}
$$

Now, we will consider the following neutral impulsive ratio-dependent predator-prey system with Holling type III functional response:

$$
\begin{gathered}
x^{\prime}(t) \\
=x(t)\left[a(t)-b(t) x\left(t-\sigma_{1}(t)\right)-\rho(t) x^{\prime}\left(t-\sigma_{2}(t)\right)\right] \\
\quad-\frac{c(t) x^{2}(t)}{m^{2}(t) y^{2}(t)+x^{2}(t)} y(t) \\
y^{\prime}(t) \quad \\
=y(t)\left[\begin{array}{c}
\left.d(t)+\frac{e(t) x^{2}\left(t-\sigma_{3}(t)\right)}{m^{2}(t) y^{2}\left(t-\sigma_{3}(t)\right)+x^{2}\left(t-\sigma_{3}(t)\right)}\right] \\
t \neq t_{k}, \quad k=1,2, \ldots, \\
x\left(t_{k}^{+}\right)-x\left(t_{k}\right)=\theta_{1 k} x\left(t_{k}\right), \\
y\left(t_{k}^{+}\right)-y\left(t_{k}\right)=\theta_{2 k} y\left(t_{k}\right), \\
t=t_{k}, \quad k=1,2, \ldots,
\end{array}\right.
\end{gathered}
$$

where $x$ and $y$ represent the prey and predator densities; $a$ is the intrinsic growth rate of the prey; $b$ is the densitydependent coefficient of the prey; $d$ is the death rate of the predator; $\theta_{1 k}, \theta_{2 k}\left(k \in N^{+}=\{1,2, \ldots\}\right)$ are the regular pulses at time $t_{k}$ of the prey and predator, respectively. In addition, $x\left(t_{k}^{+}\right), x\left(t_{k}^{-}\right)$represent the right and left limits of $x(t)$ at $t_{k}$; $y\left(t_{k}^{+}\right), y\left(t_{k}^{-}\right)$represent the right and left limits of $y(t)$ at $t_{k}$.

Throughout the paper, we give the hypothesis as follows:

$\left(\mathrm{H}_{1}\right)\left(b, c, e, m, \rho \in C\left(R_{+},(0,+\infty)\right), a, d \in C\left(R_{+}, R\right)\right.$, $\sigma_{1}, \sigma_{3} \in C^{1}\left(R_{+}, R\right), \sigma_{2} \in C^{2}\left(R_{+}, R\right)$ are $\omega$-periodic functions; $\int_{0}^{\omega} a(t) \mathrm{d} t>0, \int_{0}^{\omega} d(t) \mathrm{d} t>0 ; 0<t_{1}<t_{2}<$ $\cdots t_{k}<\cdots$ are fixed points and $\lim _{k \rightarrow \infty} t_{k}=+\infty$;

$\left(\mathrm{H}_{2}\right) \theta_{i k}>-1, i=1,2 ; k \in N^{+} ; \prod_{0<t_{k}<t}\left(1+\theta_{i k}\right)$ is $\omega$ periodic function for $i=1,2$.

The rest of this paper is organized as follows. We first introduce a lemma and the continuation theorem. Then, in Section 3, we obtain the existence of periodic solutions for system (3). In Section 4, a conclusion is given.

\section{Preliminaries}

In view of the actual applications of (3), we will only consider the solutions of system (3) with the initial condition:

$$
\begin{aligned}
& x(t)=\varphi(t), \quad \varphi \in C^{1}([-\sigma, 0],[0, \infty)), \varphi(0)>0, \\
& y(t)=\psi(t), \quad \psi \in C^{1}([-\sigma, 0],[0, \infty)), \psi(0)>0,
\end{aligned}
$$

where $\sigma=\max _{t \in[0, \omega]}\left\{\sigma_{1}(t), \sigma_{2}(t), \sigma_{3}(t)\right\}$.

Definition 1. Assume that $(x, y)^{T} \in C([-\sigma, \infty),(0,+\infty) \times$ $(0,+\infty))$ satisfies the initial condition (4). Moreover,

(i) $x$ and $y$ are absolutely continuous on each interval $\left(0, t_{1}\right]$ and $\left(t_{k}, t_{k+1}\right], k \in N^{+}$;

(ii) for $k \in N^{+},\left(x\left(t_{k}^{+}\right), y\left(t_{k}^{+}\right)\right)^{T}$ and $\left(x\left(t_{k}^{-}\right), y\left(t_{k}^{-}\right)\right)^{T}$ exist and $\left(x\left(t_{k}^{-}\right), y\left(t_{k}^{-}\right)\right)^{T}=\left(x\left(t_{k}\right), y\left(t_{k}\right)\right)^{T}$;

(iii) for $k \in N^{+},(x, y)^{T}$ satisfies (3) for almost everywhere in $[0, \infty) \backslash\left\{t_{k}\right\}$.

Then $(x, y)^{T}$ is a positive solution of the initial value problem (3) and (4) on $[-\sigma, \infty)$.

Consider the following system:

$$
\begin{gathered}
p^{\prime}(t)=p(t)\left[a(t)-B(t) p\left(t-\sigma_{1}(t)\right)-\delta(t) p^{\prime}\left(t-\sigma_{2}(t)\right)\right. \\
\left.-\frac{C(t) p(t) q(t)}{M_{1}^{2}(t) q^{2}(t)+N_{1}^{2}(t) p^{2}(t)}\right],
\end{gathered}
$$

$q^{\prime}(t)$

$$
\begin{aligned}
= & q(t) \\
& \cdot\left[-d(t)+\frac{E(t) p^{2}\left(t-\sigma_{3}(t)\right)}{M_{2}^{2}(t) q^{2}\left(t-\sigma_{3}(t)\right)+N_{2}^{2}(t) p^{2}\left(t-\sigma_{3}(t)\right)}\right],
\end{aligned}
$$

where

$$
\begin{gathered}
B(t)=b(t) \prod_{0<t_{k}<t-\sigma_{1}(t)}\left(1+\theta_{1 k}\right), \\
\delta(t)=\rho(t) \prod_{0<t_{k}<t-\sigma_{2}(t)}\left(1+\theta_{1 k}\right), \\
C(t)=c(t) \prod_{0<t_{k}<t}\left(1+\theta_{1 k}\right)\left(1+\theta_{2 k}\right), \\
M_{1}(t)=m(t) \prod_{0<t_{k}<t}\left(1+\theta_{2 k}\right), \\
N_{1}(t)=\prod_{0<t_{k}<t}\left(1+\theta_{1 k}\right),
\end{gathered}
$$




$$
\begin{gathered}
E(t)=e(t) \prod_{0<t_{k}<t-\sigma_{3}(t)}\left(1+\theta_{1 k}\right)^{2}, \\
M_{2}(t)=m(t) \prod_{0<t_{k}<t-\sigma_{3}(t)}\left(1+\theta_{2 k}\right), \\
N_{2}(t)=\prod_{0<t_{k}<t-\sigma_{3}(t)}\left(1+\theta_{1 k}\right) .
\end{gathered}
$$

By $\left(\mathrm{H}_{1}\right)$ and $\left(\mathrm{H}_{2}\right)$, the above functions are all positive $\omega$ periodic functions.
Lemma 2. Assume that $\left(H_{1}\right)$ and $\left(H_{2}\right)$ hold. If $(p, q)^{T}$ is a positive solution of system (5), then $(x, y)^{T}$ is a positive solution of system (3), where

$$
x(t)=p(t) \prod_{0<t_{k}<t}\left(1+\theta_{1 k}\right), \quad y(t)=q(t) \prod_{0<t_{k}<t}\left(1+\theta_{2 k}\right) .
$$

Proof. It is easy to see that $x$ and $y$ are positive and absolutely continuous on each interval $\left(t_{k}, t_{k+1}\right]$. For $t \neq t_{k}, k \in N^{+}$,

$$
\begin{aligned}
& x^{\prime}(t)-x(t)\left[a(t)-b(t) x\left(t-\sigma_{1}(t)\right)-\rho(t) x^{\prime}\left(t-\sigma_{2}(t)\right)-\frac{c(t) x(t) y(t)}{m^{2}(t) y^{2}(t)+x^{2}(t)}\right] \\
& =p^{\prime}(t) \prod_{0<t_{k}<t}\left(1+\theta_{1 k}\right) \\
& -p(t) \prod_{0<t_{k}<t}\left(1+\theta_{1 k}\right) \\
& \cdot\left[a(t)-b(t) p\left(t-\sigma_{1}(t)\right) \prod_{0<t_{k}<t-\sigma_{1}(t)}\left(1+\theta_{1 k}\right)-\rho(t) p^{\prime}\left(t-\sigma_{2}(t)\right) \prod_{0<t_{k}<t-\sigma_{2}(t)}\left(1+\theta_{1 k}\right)\right. \\
& \left.-\frac{c(t) p(t) q(t) \prod_{0<t_{k}<t}\left(1+\theta_{1 k}\right)\left(1+\theta_{2 k}\right)}{m^{2}(t) q^{2}(t) \prod_{0<t_{k}<t}\left(1+\theta_{2 k}\right)^{2}+p^{2}(t) \prod_{0<t_{k}<t}\left(1+\theta_{1 k}\right)^{2}}\right] \\
& =\prod_{0<t_{k}<t}\left(1+\theta_{1 k}\right)\left\{p^{\prime}(t)-p(t)\left[a(t)-b(t) p\left(t-\sigma_{1}(t)\right) \prod_{0<t_{k}<t-\sigma_{1}(t)}\left(1+\theta_{1 k}\right)\right.\right. \\
& -\rho(t) p^{\prime}\left(t-\sigma_{2}(t)\right) \prod_{0<t_{k}<t-\sigma_{2}(t)}\left(1+\theta_{1 k}\right) \\
& \left.\left.-\frac{c(t) p(t) q(t) \prod_{0<t_{k}<t}\left(1+\theta_{1 k}\right)\left(1+\theta_{2 k}\right)}{m^{2}(t) q^{2}(t) \prod_{0<t_{k}<t}\left(1+\theta_{2 k}\right)^{2}+p^{2}(t) \prod_{0<t_{k}<t}\left(1+\theta_{1 k}\right)^{2}}\right]\right\} \\
& =\prod_{0<t_{k}<t}\left(1+\theta_{1 k}\right)\left\{p^{\prime}(t)-p(t)\left[a(t)-B(t) p\left(t-\sigma_{1}(t)\right)-\delta(t) p^{\prime}\left(t-\sigma_{2}(t)\right)\right.\right. \\
& \left.\left.-\frac{C(t) p(t) q(t)}{M_{1}^{2}(t) q^{2}(t)+N_{1}^{2}(t) p^{2}(t)}\right]\right\} \\
& =0 \text {. }
\end{aligned}
$$

Hence, the first equation of system (3) holds. Similarly

$$
\begin{aligned}
& y^{\prime}(t)-y(t)\left[-d(t)+\frac{e(t) x^{2}\left(t-\sigma_{3}(t)\right)}{m^{2}(t) y^{2}\left(t-\sigma_{3}(t)\right)+x^{2}\left(t-\sigma_{3}(t)\right)}\right] \\
& =q^{\prime}(t) \prod_{0<t_{k}<t}\left(1+\theta_{2 k}\right) \\
& -q(t) \prod_{0<t_{k}<t}\left(1+\theta_{2 k}\right)\left[-d(t)+\frac{e(t) p^{2}\left(t-\sigma_{3}(t)\right) \prod_{0<t_{k}<t-\sigma_{3}(t)}\left(1+\theta_{1 k}\right)^{2}}{m^{2}(t) q^{2}\left(t-\sigma_{3}(t)\right) \prod_{0<t_{k}<t-\sigma_{3}(t)}\left(1+\theta_{2 k}\right)^{2}+p^{2}\left(t-\sigma_{3}(t)\right) \prod_{0<t_{k}<t-\sigma_{3}(t)}\left(1+\theta_{1 k}\right)^{2}}\right]
\end{aligned}
$$




$$
\begin{aligned}
& =\prod_{0<t_{k}<t}\left(1+\theta_{2 k}\right)\left\{q^{\prime}(t)-q(t)\right. \\
& \left.\cdot\left[-d(t)+\frac{e(t) p^{2}\left(t-\sigma_{3}(t)\right) \prod_{0<t_{k}<t-\sigma_{3}(t)}\left(1+\theta_{1 k}\right)^{2}}{m^{2}(t) q^{2}\left(t-\sigma_{3}(t)\right) \prod_{0<t_{k}<t-\sigma_{3}(t)}\left(1+\theta_{2 k}\right)^{2}+p^{2}\left(t-\sigma_{3}(t)\right) \prod_{0<t_{k}<t-\sigma_{3}(t)}\left(1+\theta_{1 k}\right)^{2}}\right]\right\} \\
& =\prod_{0<t_{k}<t}\left(1+\theta_{2 k}\right)\left\{q^{\prime}(t)-q(t)\left[-d(t)+\frac{E(t) p^{2}\left(t-\sigma_{3}(t)\right)}{M_{2}^{2}(t) q^{2}\left(t-\sigma_{3}(t)\right)+N_{2}^{2}(t) p^{2}\left(t-\sigma_{3}(t)\right)}\right]\right\} . \\
& =0 .
\end{aligned}
$$

That is, the second equation of system (3) holds. For $t=t_{k}$, $k \in N^{+}$,

$$
\begin{aligned}
& x\left(t_{k}^{+}\right)=\lim _{t \rightarrow t_{k}^{+}}\left\{p(t) \prod_{0<t_{j}<t}\left(1+\theta_{1 j}\right)\right\} \\
&=p\left(t_{k}\right) \prod_{0<t_{j} \leq t_{k}}\left(1+\theta_{1 j}\right), \\
& y\left(t_{k}^{+}\right)=\lim _{t \rightarrow t_{k}^{+}}\left\{q(t) \prod_{0<t_{j}<t}\left(1+\theta_{2 j}\right)\right\} \\
&=q\left(t_{k}\right) \prod_{0<t_{j} \leq t_{k}}\left(1+\theta_{2 j}\right), \\
& x \\
& x\left(t_{k}\right)=p\left(t_{k}\right) \prod_{0<t_{j}<t_{k}}\left(1+\theta_{1 j}\right), \\
& y\left(t_{k}\right)=q\left(t_{k}\right) \prod_{0<t_{j}<t_{k}}\left(1+\theta_{2 j}\right) .
\end{aligned}
$$

Hence, for $t=t_{k}, k \in N^{+}$,

$$
x\left(t_{k}^{+}\right)=\left(1+\theta_{1 k}\right) x\left(t_{k}\right), \quad y\left(t_{k}^{+}\right)=\left(1+\theta_{2 k}\right) y\left(t_{k}\right) .
$$

So $(x, y)^{T}$ is a positive solution of system (3).

Next, we recall some concepts and results on coincidence degree.

Let $X, Z$ be normed vector spaces; let $L: \operatorname{Dom} L \subset X \rightarrow$ $Z$ be a linear mapping. Assume that $\operatorname{dim} \operatorname{Ker} L=\operatorname{codim} \operatorname{Im} L<$ $+\infty$ and $\operatorname{Im} L$ is closed in $Z$; then the mapping $L$ is said to be a Fredholm mapping of index zero.

If $L$ is a Fredholm mapping of index zero, then there exist continuous projectors $P: X \rightarrow X$ and $Q: Z \rightarrow Z$ satisfying $\operatorname{Im} P=\operatorname{Ker} L, \operatorname{Ker} Q=\operatorname{Im} L=\operatorname{Im}(I-Q)$. It is obvious that the restriction $L_{P}$ of $L$ to $\operatorname{Dom} L \cap \operatorname{Ker} P:(I-P) X \rightarrow \operatorname{Im} L$ has an inverse function. Denote $K_{P}: \operatorname{Im} L \rightarrow$ Dom $L$ by $K_{P}=L_{P}^{-1}$.

Let $N: X \rightarrow Z$ be a continuous mapping. $N$ is $L$ compact on $\bar{\Omega}$; if $\Omega$ is an open bounded subset of $X, Q N(\bar{\Omega})$ is bounded and $K_{P}(I-Q) N: \bar{\Omega} \rightarrow X$ is relatively compact.

Lemma 3 (see [14]). Let $\Omega \subset X$ be an open bounded set; $L$ be a Fredholm mapping of index zero; and $N$ be L-compact on $\bar{\Omega}$. If the following conditions hold:

(i) $L x \neq \lambda N x$, for any $\lambda \in(0,1), x \in \partial \Omega \cap \operatorname{Dom} L$;

(ii) $Q N x \neq 0$, for any $x \in \partial \Omega \cap \operatorname{Ker} L$;

(iii) $\operatorname{deg}\{J Q N, \Omega \cap \operatorname{Ker} L, 0\} \neq 0$, where $J: \operatorname{Im} Q \rightarrow \operatorname{Ker} L$ is an isomorphism;

then $L x=N x$ has at least one solution in $\bar{\Omega} \cap \operatorname{Dom} L$.

\section{Main Results}

Let $h$ be a continuous $\omega$-periodic function. Denote

$$
\begin{gathered}
|h|_{0}=\max _{t \in[0, \omega]}\{|h(t)|\}, \quad \bar{h}=\frac{1}{\omega} \int_{0}^{\omega} h(t) \mathrm{d} t, \\
\widehat{h}=\frac{1}{\omega} \int_{0}^{\omega}|h(t)| \mathrm{d} t .
\end{gathered}
$$

In the following, we will present our main results.

Theorem 4. Suppose that $\left(H_{1}\right),\left(H_{2}\right)$, and the following conditions hold.

$\left(\mathrm{H}_{3}\right)$ For $t \in[0, \infty), \sigma_{1}^{\prime}(t)<1, \sigma_{2}^{\prime}(t)<1$; let $t=\phi_{1}(p)$ be the inverse function of $p=t-\sigma_{1}(t)$ and $G(t)>H^{\prime}(t)$, where $G(t)=B\left(\phi_{1}\left(t-\sigma_{2}(t)\right)\right)\left(1-\sigma_{2}^{\prime}(t)\right) /\left(1-\sigma_{1}^{\prime}\left(\phi_{1}(t-\right.\right.$ $\left.\left.\left.\sigma_{2}(t)\right)\right)\right)$ and $H(t)=\delta(t) /\left(1-\sigma_{2}^{\prime}(t)\right)$. 
$\left(\mathrm{H}_{4}\right)$ Consider that $|\delta|_{0} e^{A_{1}}<1$, where $A_{1}=$ $\ln \left[2 \bar{a} \max _{t \in[0, \omega]}\left\{\left(1-\sigma_{2}^{\prime}(t)\right) /\left(G(t)-H^{\prime}(t)\right)\right\}\right]+$ $2 \bar{a}|H|_{0} \max _{t \in[0, \omega]}\left\{1 /\left(G(t)-H^{\prime}(t)\right)\right\}+(\widehat{a}+\bar{a}) \omega$.

$\left(\mathrm{H}_{5}\right)$ Consider that $2 \bar{a}>\bar{C}\left|1 / M_{1} N_{1}\right|_{0}$ and $\bar{E}>\bar{d}\left|N_{2}^{2}\right|_{0}$.

Then system (3) has at least one positive $\omega$-periodic solution. Proof. Construct the system

$$
\begin{gathered}
u_{1}^{\prime}(t)=a(t)-B(t) e^{u_{1}\left(t-\sigma_{1}(t)\right)} \\
-\delta(t) e^{u_{1}\left(t-\sigma_{2}(t)\right)} u_{1}^{\prime}\left(t-\sigma_{2}(t)\right) \\
-\frac{C(t) e^{u_{1}(t)} e^{u_{2}(t)}}{M_{1}^{2}(t) e^{2 u_{2}(t)}+N_{1}^{2}(t) e^{2 u_{1}(t)}}, \\
u_{2}^{\prime}(t)=-d(t)+\frac{E(t) e^{2 u_{1}\left(t-\sigma_{3}(t)\right)}}{M_{2}^{2}(t) e^{2 u_{2}\left(t-\sigma_{3}(t)\right)}+N_{2}^{2}(t) e^{2 u_{1}\left(t-\sigma_{3}(t)\right)}} .
\end{gathered}
$$

Apparently, if there exists one $\omega$-periodic solution $\left(u_{1}^{*}(t)\right.$, $\left.u_{2}^{*}(t)\right)^{T}$ for system (13), then $\left(p^{*}(t), q^{*}(t)\right)^{T}=\left(e^{u_{1}^{*}(t)}, e^{u_{2}^{*}(t)}\right)^{T}$ is a positive $\omega$-periodic solution of system (5). By $\left(\mathrm{H}_{2}\right)$ and Lemma 2, Theorem 4 holds if we can show that there exists one $\omega$-periodic solution of system (13).

Let $Z$ be the real Banach space $\left\{u=\left(u_{1}, u_{2}\right)^{T} \in C\left(R, R^{2}\right)\right.$ : $\left.u_{i}(t+\omega)=u_{i}(t), t \in R, i=1,2\right\}$ with the norm

$$
|u|_{\infty}=\max _{t \in[0, \omega]}\left\{\left|u_{1}(t)\right|+\left|u_{2}(t)\right|\right\} .
$$

Let $X$ be the real Banach space $\left\{u=\left(u_{1}, u_{2}\right)^{T} \in C^{1}\left(R, R^{2}\right)\right.$ : $\left.u_{i}(t+\omega)=u_{i}(t), t \in R, i=1,2\right\}$ with the norm

$$
\|u\|=|u|_{\infty}+\left|u^{\prime}\right|_{\infty} .
$$

Define $L: X \rightarrow Z$ and $N: X \rightarrow Z$ by

$$
\begin{gathered}
L\left(u_{1}(t), u_{2}(t)\right)^{T}=\left(u_{1}^{\prime}(t), u_{2}^{\prime}(t)\right)^{T}, \\
N\left[\begin{array}{l}
u_{1}(t) \\
u_{2}(t)
\end{array}\right]=\left[\begin{array}{c}
a(t)-B(t) e^{u_{1}\left(t-\sigma_{1}(t)\right)}-\delta(t) e^{u_{1}\left(t-\sigma_{2}(t)\right)} u_{1}^{\prime}\left(t-\sigma_{2}(t)\right)-\frac{C(t) e^{u_{1}(t)} e^{u_{2}(t)}}{M_{1}^{2}(t) e^{2 u_{2}(t)}+N_{1}^{2}(t) e^{2 u_{1}(t)}} \\
-d(t)+\frac{E(t) e^{2 u_{1}\left(t-\sigma_{3}(t)\right)}}{M_{2}^{2}(t) e^{2 u_{2}\left(t-\sigma_{3}(t)\right)}+N_{2}^{2}(t) e^{2 u_{1}\left(t-\sigma_{3}(t)\right)}}
\end{array}\right] .
\end{gathered}
$$

Therefore, system (13) can be written as

$$
L u=N u, \quad u \in X .
$$

Evidently, $\operatorname{Ker} L=R^{2}, \operatorname{Im} L=\left\{\left(u_{1}, u_{2}\right)^{T} \in Z: \int_{0}^{\omega} u_{i}(t) \mathrm{d} t=0\right.$, $i=1,2\}$ is closed in $Z$, and $\operatorname{dimKer} L=\operatorname{codim} \operatorname{Im} L=2$. Hence $L$ is a Fredholm mapping of index zero.

Let $P: X \rightarrow X$ and $Q: Z \rightarrow Z$ be

$$
\begin{aligned}
& P\left[\begin{array}{l}
u_{1}(t) \\
u_{2}(t)
\end{array}\right]=\left[\begin{array}{l}
\bar{u}_{1} \\
\bar{u}_{2}
\end{array}\right], \quad\left[\begin{array}{l}
u_{1} \\
u_{2}
\end{array}\right] \in X, \\
& Q\left[\begin{array}{l}
u_{1}(t) \\
u_{2}(t)
\end{array}\right]=\left[\begin{array}{l}
\bar{u}_{1} \\
\bar{u}_{2}
\end{array}\right], \quad\left[\begin{array}{l}
u_{1} \\
u_{2}
\end{array}\right] \in Z .
\end{aligned}
$$

The generalized inverse (to $L$ ) $K_{P}: \operatorname{Im} L \rightarrow \operatorname{Ker} P \cap \operatorname{Dom} L$ is

$$
K_{P}(u)=\int_{0}^{t} u(s) \mathrm{d} s-\frac{1}{\omega} \int_{0}^{\omega} \int_{0}^{t} u(s) \mathrm{d} s \mathrm{~d} t .
$$

Clearly, $P$ and $Q$ are continuous projectors satisfying

$$
\operatorname{Im} P=\operatorname{Ker} L, \quad \operatorname{Ker} Q=\operatorname{Im} L=\operatorname{Im}(I-Q) .
$$

$$
(\mathrm{QN}) u=\left[\begin{array}{c}
\frac{1}{\omega} \int_{0}^{\omega}\left[a(t)-B(t) e^{u_{1}\left(t-\sigma_{1}(t)\right)}+H^{\prime}(t) e^{u_{1}\left(t-\sigma_{2}(t)\right)}-\frac{C(t) e^{u_{1}(t)} e^{u_{2}(t)}}{M_{1}^{2}(t) e^{2 u_{2}(t)}+N_{1}^{2}(t) e^{2 u_{1}(t)}}\right] \mathrm{d} t \\
\frac{1}{\omega} \int_{0}^{\omega}\left[-d(t)+\frac{E(t) e^{2 u_{1}\left(t-\sigma_{3}(t)\right)}}{M_{2}^{2}(t) e^{2 u_{2}\left(t-\sigma_{3}(t)\right)}+N_{2}^{2}(t) e^{2 u_{1}\left(t-\sigma_{3}(t)\right)}}\right] \mathrm{d} t
\end{array}\right],
$$




$$
\begin{aligned}
&\left(K_{P}(I-Q) N\right) u {\left[\begin{array}{c}
\left.\int_{0}^{t}\left[a(s)-B(s) e^{u_{1}\left(s-\sigma_{1}(s)\right)}+H^{\prime}(s) e^{u_{1}\left(s-\sigma_{2}(s)\right)}-\frac{C(s) e^{u_{1}(s)} e^{u_{2}(s)}}{M_{1}^{2}(s) e^{2 u_{2}(s)}+N_{1}^{2}(s) e^{2 u_{1}(s)}}\right] \mathrm{d} s\right] \\
-H(t) e^{u_{1}\left(t-\sigma_{2}(t)\right)}+H(0) e^{u_{1}\left(-\sigma_{2}(0)\right)}
\end{array}\right] \mathrm{d} } \\
& \int_{0}^{t}\left[-d(s)+\frac{E(s) e^{2 u_{1}\left(s-\sigma_{3}(s)\right)}}{M_{2}^{2}(s) e^{2 u_{2}\left(s-\sigma_{3}(s)\right)}+N_{2}^{2}(s) e^{2 u_{1}\left(s-\sigma_{3}(s)\right)}}\right] \\
&-\left[\begin{array}{c}
\frac{1}{\omega} \int_{0}^{\omega} \int_{0}^{t}\left[a(s)-B(s) e^{u_{1}\left(s-\sigma_{1}(s)\right)}+H^{\prime}(s) e^{u_{1}\left(s-\sigma_{2}(s)\right)}-\frac{C(s) e^{u_{1}(s)} e^{u_{2}(s)}}{M_{1}^{2}(s) e^{2 u_{2}(s)}+N_{1}^{2}(s) e^{2 u_{1}(s)}}\right] \mathrm{d} \mathrm{d} t \\
-\frac{1}{\omega} \int_{0}^{\omega}\left[H(t) e^{u_{1}\left(t-\sigma_{2}(t)\right)}-H(0) e^{u_{1}\left(-\sigma_{2}(0)\right)}\right] \mathrm{d} t
\end{array}\right] \mathrm{d} \mathrm{d} t \\
& \frac{1}{\omega} \int_{0}^{\omega} \int_{0}^{t}\left[-d(s)+\frac{E(s) e^{2 u_{1}\left(s-\sigma_{3}(s)\right)}}{M_{2}^{2}(s) e^{2 u_{2}\left(s-\sigma_{3}(s)\right)}+N_{2}^{2}(s) e^{2 u_{1}\left(s-\sigma_{3}(s)\right)}}\right] \\
&-
\end{aligned}
$$

Obviously, it is easy to check that $Q N$ and $K_{P}(I-Q) N$ are continuous. Furthermore, $Q N(\bar{\Omega})$ is bounded; $K_{P}(I-$ Q) $N(\bar{\Omega})$ are relatively compact for any open bounded set $\Omega \subset X$. Thus $N$ is $L$-compact on $\bar{\Omega}$.

For any $\lambda \in(0,1)$, consider $L u=\lambda N u$; that is,

$$
\begin{gathered}
u_{1}^{\prime}(t)=\lambda\left[a(t)-B(t) e^{u_{1}\left(t-\sigma_{1}(t)\right)}-\delta(t) e^{u_{1}\left(t-\sigma_{2}(t)\right)}\right. \\
\left.\cdot u_{1}^{\prime}\left(t-\sigma_{2}(t)\right)-\frac{C(t) e^{u_{1}(t)} e^{u_{2}(t)}}{M_{1}^{2}(t) e^{2 u_{2}(t)}+N_{1}^{2}(t) e^{2 u_{1}(t)}}\right], \\
u_{2}^{\prime}(t)=\lambda\left[-d(t)+\frac{E(t) e^{2 u_{1}\left(t-\sigma_{3}(t)\right)}}{M_{2}^{2}(t) e^{2 u_{2}\left(t-\sigma_{3}(t)\right)}+N_{2}^{2}(t) e^{2 u_{1}\left(t-\sigma_{3}(t)\right)}}\right] .
\end{gathered}
$$

Let $\left(u_{1}, u_{2}\right)^{T} \in X$ be a solution of (22). Thus

$$
\begin{gathered}
\int_{0}^{\omega}\left[a(t)-B(t) e^{u_{1}\left(t-\sigma_{1}(t)\right)}\right. \\
-\delta(t) e^{u_{1}\left(t-\sigma_{2}(t)\right)} u_{1}^{\prime}\left(t-\sigma_{2}(t)\right) \\
\left.-\frac{C(t) e^{u_{1}(t)} e^{u_{2}(t)}}{M_{1}^{2}(t) e^{2 u_{2}(t)}+N_{1}^{2}(t) e^{2 u_{1}(t)}}\right] \mathrm{d} t=0, \\
\int_{0}^{\omega}\left[-d(t)+\frac{E(t) e^{2 u_{1}\left(t-\sigma_{3}(t)\right)}}{M_{2}^{2}(t) e^{2 u_{2}\left(t-\sigma_{3}(t)\right)}+N_{2}^{2}(t) e^{2 u_{1}\left(t-\sigma_{3}(t)\right)}}\right] \mathrm{d} t=0 .
\end{gathered}
$$

By $\left(\mathrm{H}_{3}\right)$,

$$
\begin{gathered}
\int_{0}^{\omega} \delta(t) e^{u_{1}\left(t-\sigma_{2}(t)\right)} u_{1}^{\prime}\left(t-\sigma_{2}(t)\right) \mathrm{d} t \\
=\int_{0}^{\omega} H(t)\left(e^{u_{1}\left(t-\sigma_{2}(t)\right.}\right)^{\prime} \mathrm{d} t \\
=-\int_{0}^{\omega} H^{\prime}(t) e^{u_{1}\left(t-\sigma_{2}(t)\right)} \mathrm{d} t .
\end{gathered}
$$

By (23),

$$
\begin{aligned}
& \int_{0}^{\omega}\left[B(t) e^{u_{1}\left(t-\sigma_{1}(t)\right)}-H^{\prime}(t) e^{u_{1}\left(t-\sigma_{2}(t)\right)}\right. \\
& \left.\quad+\frac{C(t) e^{u_{1}(t)} e^{u_{2}(t)}}{M_{1}^{2}(t) e^{2 u_{2}(t)}+N_{1}^{2}(t) e^{2 u_{1}(t)}}\right] \mathrm{d} t=\bar{a} \omega .
\end{aligned}
$$

By (24),

$$
\int_{0}^{\omega} \frac{E(t) e^{2 u_{1}\left(t-\sigma_{3}(t)\right)}}{M_{2}^{2}(t) e^{2 u_{2}\left(t-\sigma_{3}(t)\right)}+N_{2}^{2}(t) e^{2 u_{1}\left(t-\sigma_{3}(t)\right)}} \mathrm{d} t=\bar{d} \omega .
$$

Set $t=\phi_{2}(p)$ be the inverse function of $p=t-\sigma_{2}(t)$. Clearly, $B\left(\phi_{1}(p)\right), \sigma_{1}^{\prime}\left(\phi_{1}(p)\right), B\left(\phi_{1}\left(p-\sigma_{2}(p)\right)\right), \sigma_{1}^{\prime}\left(\phi_{1}\left(p-\sigma_{2}(p)\right)\right)$ are all $\omega$-periodic functions. In addition, $\phi_{2}(p+\omega)=\phi_{2}(p)+\omega$. By $\left(\mathrm{H}_{3}\right)$,

$$
\begin{aligned}
\int_{0}^{\omega} & B(t) e^{u_{1}\left(t-\sigma_{1}(t)\right)} \mathrm{d} t \\
\quad= & \int_{-\sigma_{1}(0)}^{\omega-\sigma_{1}(\omega)} \frac{B\left(\phi_{1}(p)\right)}{1-\sigma_{1}^{\prime}\left(\phi_{1}(p)\right)} e^{u_{1}(p)} \mathrm{d} p
\end{aligned}
$$




$$
\begin{aligned}
& =\int_{0}^{\omega} \frac{B\left(\phi_{1}(s)\right)}{1-\sigma_{1}^{\prime}\left(\phi_{1}(s)\right)} e^{u_{1}(s)} \mathrm{d} s \\
& =\int_{\phi_{2}(0)}^{\phi_{2}(\omega)} \frac{B\left(\phi_{1}\left(t-\sigma_{2}(t)\right)\right)\left(1-\sigma_{2}^{\prime}(t)\right)}{1-\sigma_{1}^{\prime}\left(\phi_{1}\left(t-\sigma_{2}(t)\right)\right)} e^{u_{1}\left(t-\sigma_{2}(t)\right)} \mathrm{d} t \\
& =\int_{\phi_{2}(0)}^{\phi_{2}(0)+\omega} G(t) e^{u_{1}\left(t-\sigma_{2}(t)\right)} \mathrm{d} t \\
& =\int_{0}^{\omega} G(t) e^{u_{1}\left(t-\sigma_{2}(t)\right)} \mathrm{d} t .
\end{aligned}
$$

Thus

$$
\begin{aligned}
& \int_{0}^{\omega}\left[\left(G(t)-H^{\prime}(t)\right) e^{u_{1}\left(t-\sigma_{2}(t)\right)}\right. \\
& \left.\quad+\frac{C(t) e^{u_{1}(t)} e^{u_{2}(t)}}{M_{1}^{2}(t) e^{2 u_{2}(t)}+N_{1}^{2}(t) e^{2 u_{1}(t)}}\right] \mathrm{d} t=\bar{a} \omega .
\end{aligned}
$$

$\leq \int_{0}^{\omega}|a(t)| \mathrm{d} t$

$$
\begin{gathered}
+\int_{0}^{\omega}\left[\left(G(t)-H^{\prime}(t)\right) e^{u_{1}\left(t-\sigma_{2}(t)\right)}\right. \\
\left.+\frac{C(t) e^{u_{1}(t)} e^{u_{2}(t)}}{M_{1}^{2}(t) e^{2 u_{2}(t)}+N_{1}^{2}(t) e^{2 u_{1}(t)}}\right] \mathrm{d} t \\
=(\widehat{a}+\bar{a}) \omega .
\end{gathered}
$$

Note that $G\left(\phi_{2}(p)\right), H^{\prime}\left(\phi_{2}(p)\right), \sigma_{2}^{\prime}\left(\phi_{2}(p)\right)$ are all $\omega$-periodic functions. By (29) and $\left(\mathrm{H}_{3}\right)$,

$$
\begin{aligned}
\bar{a} \omega & \geq \int_{0}^{\omega}\left[G(t)-H^{\prime}(t)\right] e^{u_{1}\left(t-\sigma_{2}(t)\right)} \mathrm{d} t \\
& =\int_{-\sigma_{2}(0)}^{\omega-\sigma_{2}(\omega)} \frac{G\left(\phi_{2}(p)\right)-H^{\prime}\left(\phi_{2}(p)\right)}{1-\sigma_{2}^{\prime}\left(\phi_{2}(p)\right)} e^{u_{1}(p)} \mathrm{d} p \\
& =\int_{0}^{\omega} \frac{G\left(\phi_{2}(t)\right)-H^{\prime}\left(\phi_{2}(t)\right)}{1-\sigma_{2}^{\prime}\left(\phi_{2}(t)\right)} e^{u_{1}(t)} \mathrm{d} t,
\end{aligned}
$$

So there exists $\xi \in[0, \omega]$ satisfying

$$
\begin{aligned}
& \left(G(\xi)-H^{\prime}(\xi)\right) e^{u_{1}\left(\xi-\sigma_{2}(\xi)\right)} \\
& \quad+\frac{G\left(\phi_{2}(\xi)\right)-H^{\prime}\left(\phi_{2}(\xi)\right)}{1-\sigma_{2}^{\prime}\left(\phi_{2}(\xi)\right)} e^{u_{1}(\xi)} \leq 2 \bar{a} .
\end{aligned}
$$

By $\left(\mathrm{H}_{3}\right)$,

$$
\begin{aligned}
& u_{1}(\xi) \leq \ln \left[2 \bar{a} \max _{t \in[0, \omega]}\left\{\frac{1-\sigma_{2}^{\prime}(t)}{G(t)-H^{\prime}(t)}\right\}\right], \\
& e^{u_{1}\left(\xi-\sigma_{2}(\xi)\right)} \leq 2 \bar{a} \max _{t \in[0, \omega]}\left\{\frac{1}{G(t)-H^{\prime}(t)}\right\} .
\end{aligned}
$$

By $(30)$ and $\left(\mathrm{H}_{4}\right)$, for $t \in[0, \omega]$,

$$
\begin{aligned}
& u_{1}(t)+\lambda H(t) e^{u_{1}\left(t-\sigma_{2}(t)\right)} \\
& \leq u_{1}(\xi)+\lambda H(\xi) e^{u_{1}\left(\xi-\sigma_{2}(\xi)\right)} \\
&+\int_{0}^{\omega}\left|\frac{\mathrm{d}}{\mathrm{d} t}\left[u_{1}(t)+\lambda H(t) e^{u_{1}\left(t-\sigma_{2}(t)\right)}\right]\right| \mathrm{d} t \\
& \leq \ln \left[2 \bar{a} \max _{t \in[0, \omega]}\left\{\frac{1-\sigma_{2}^{\prime}(t)}{G(t)-H^{\prime}(t)}\right\}\right] \\
&+2 \bar{a}|H|_{0} \max _{t \in[0, \omega]}\left\{\frac{1}{G(t)-H^{\prime}(t)}\right\}+(\widehat{a}+\bar{a}) \omega=A_{1} . \\
& \text { As } \lambda H(t) e^{u_{1}\left(t-\sigma_{2}(t)\right)} \geq 0, \\
& u_{1}(t) \leq A_{1}, \quad t \in[0, \omega] .
\end{aligned}
$$

By (22) and (36),

$$
\begin{aligned}
&\left|u_{1}^{\prime}(t)\right|=\lambda \mid a(t)-B(t) e^{u_{1}\left(t-\sigma_{1}(t)\right)} \\
&-\delta(t) e^{u_{1}\left(t-\sigma_{2}(t)\right)} u_{1}^{\prime}\left(t-\sigma_{2}(t)\right) \\
& \quad-\frac{C(t) e^{u_{1}(t)} e^{u_{2}(t)}}{M_{1}^{2}(t) e^{2 u_{2}(t)}+N_{1}^{2}(t) e^{2 u_{1}(t)}} \mid \\
& \leq|a|_{0}+|B|_{0} e^{A_{1}}+|\delta|_{0} e^{A_{1}}\left|u_{1}^{\prime}\right|_{0}+\left|\frac{C}{2 M_{1} N_{1}}\right|_{0}
\end{aligned}
$$

By $\left(\mathrm{H}_{4}\right)$,

$$
\left|u_{1}^{\prime}\right|_{0} \leq \frac{1}{1-|\delta|_{0} e^{A_{1}}}\left(|a|_{0}+|B|_{0} e^{A_{1}}+\left|\frac{C}{2 M_{1} N_{1}}\right|_{0}\right)=: D_{1} .
$$

In addition, there exist $\xi_{i}, \eta_{i} \in[0, \omega]$ satisfying

$$
\begin{array}{r}
u_{i}\left(\xi_{i}\right)=\min _{t \in[0, \omega]}\left\{u_{i}(t)\right\}, \\
u_{i}\left(\eta_{i}\right)=\max _{t \in[0, \omega]}\left\{u_{i}(t)\right\}, \\
i=1,2 .
\end{array}
$$

$$
\left.+\frac{G\left(\phi_{2}(t)\right)-H^{\prime}\left(\phi_{2}(t)\right)}{1-\sigma_{2}^{\prime}\left(\phi_{2}(t)\right)} e^{u_{1}(t)}\right] \mathrm{d} t \leq 2 \bar{a} \omega .
$$


By (29) and $\left(\mathrm{H}_{3}\right)$,

$$
\begin{aligned}
\bar{a} \omega & -\left(\bar{G}-\overline{H^{\prime}}\right) e^{u_{1}\left(\eta_{1}\right)} \omega \\
& \leq \bar{a} \omega-\int_{0}^{\omega}\left[\left(G(t)-H^{\prime}(t)\right) e^{u_{1}\left(t-\sigma_{2}(t)\right)}\right] \mathrm{d} t \\
& =\int_{0}^{\omega} \frac{C(t) e^{u_{1}(t)} e^{u_{2}(t)}}{M_{1}^{2}(t) e^{2 u_{2}(t)}+N_{1}^{2}(t) e^{2 u_{1}(t)}} \mathrm{d} t \\
& \leq \frac{C}{\left(\frac{C}{2 M_{1} N_{1}}\right)} \omega \leq\left|\frac{1}{2 M_{1} N_{1}}\right|_{0} \bar{C} \omega .
\end{aligned}
$$

By $\left(\mathrm{H}_{5}\right)$ and $(40)$,

$$
u_{1}\left(\eta_{1}\right) \geq \ln \left[\frac{2 \bar{a}-\left|1 / M_{1} N_{1}\right|_{0} \bar{C}}{2\left(\bar{G}-\overline{H^{\prime}}\right)}\right]
$$

By (38),

$$
\begin{aligned}
u_{1}(t) & \geq u_{1}\left(\eta_{1}\right)-\int_{0}^{\omega}\left|u_{1}^{\prime}(t)\right| \mathrm{d} t \\
& \geq \ln \left[\frac{2 \bar{a}-\left|1 / M_{1} N_{1}\right|_{0} \bar{C}}{2\left(\bar{G}-\overline{H^{\prime}}\right)}\right]-D_{1} \omega=: A_{2}, \quad t \in[0, \omega] .
\end{aligned}
$$

By (36) and (42),

$$
\left|u_{1}\right|_{0} \leq \max \left\{\left|A_{1}\right|,\left|A_{2}\right|\right\}=: A \text {. }
$$

By (27), there exists $\zeta_{1} \in[0, \omega]$ satisfying

$$
\bar{d} \omega=\frac{e^{2 u_{1}\left(\zeta_{1}-\sigma_{3}\left(\zeta_{1}\right)\right)}}{M_{2}^{2}\left(\zeta_{1}\right) e^{2 u_{2}\left(\zeta_{1}-\sigma_{3}\left(\zeta_{1}\right)\right)}+N_{2}^{2}\left(\zeta_{1}\right) e^{2 u_{1}\left(\zeta_{1}-\sigma_{3}\left(\zeta_{1}\right)\right)}} \bar{E} \omega
$$

Set $\zeta_{1}-\sigma_{3}\left(\zeta_{1}\right)=\zeta_{2}+k \omega$, where $k$ is an integer, $\zeta_{2} \in[0, \omega]$. So

$$
\bar{d} \omega=\frac{e^{2 u_{1}\left(\zeta_{2}\right)}}{M_{2}^{2}\left(\zeta_{1}\right) e^{2 u_{2}\left(\zeta_{2}\right)}+N_{2}^{2}\left(\zeta_{1}\right) e^{2 u_{1}\left(\zeta_{2}\right)}} \bar{E} \omega
$$

\section{Denote}

$$
\begin{array}{ll}
M_{2}\left(\xi_{3}\right)=\min _{t \in[0, \omega]}\left\{M_{2}(t)\right\}, & M_{2}\left(\eta_{3}\right)=\max _{t \in[0, \omega]}\left\{M_{2}(t)\right\}, \\
N_{2}\left(\xi_{4}\right)=\min _{t \in[0, \omega]}\left\{N_{2}(t)\right\}, & N_{2}\left(\eta_{4}\right)=\max _{t \in[0, \omega]}\left\{N_{2}(t)\right\} .
\end{array}
$$

Clearly, the function $x^{2} /\left(k x^{2}+l\right)$ is increasing with respect to $x$, where $k>0, l>0$. By (39), (43), (45), and (46),

$$
\begin{aligned}
\frac{\bar{d}}{\bar{E}} & \leq \frac{e^{2 u_{1}\left(\zeta_{2}\right)}}{M_{2}^{2}\left(\xi_{3}\right) e^{2 u_{2}\left(\xi_{2}\right)}+N_{2}^{2}\left(\xi_{4}\right) e^{2 u_{1}\left(\zeta_{2}\right)}} \\
& \leq \frac{e^{2 A}}{M_{2}^{2}\left(\xi_{3}\right) e^{2 u_{2}\left(\xi_{2}\right)}+N_{2}^{2}\left(\xi_{4}\right) e^{2 A}}, \\
\overline{\bar{d}} & \geq \frac{e^{2 u_{1}\left(\zeta_{2}\right)}}{M_{2}^{2}\left(\eta_{3}\right) e^{2 u_{2}\left(\eta_{2}\right)}+N_{2}^{2}\left(\eta_{4}\right) e^{2 u_{1}\left(\zeta_{2}\right)}} \\
& \geq \frac{e^{-2 A}}{M_{2}^{2}\left(\eta_{3}\right) e^{2 u_{2}\left(\eta_{2}\right)}+N_{2}^{2}\left(\eta_{4}\right) e^{-2 A}} .
\end{aligned}
$$

By $\left(\mathrm{H}_{5}\right)$,

$$
\begin{aligned}
& u_{2}\left(\xi_{2}\right) \leq A+\frac{1}{2} \ln \left(\frac{\bar{E}-\bar{d} N_{2}^{2}\left(\xi_{4}\right)}{\bar{d} M_{2}^{2}\left(\xi_{3}\right)}\right), \\
& u_{2}\left(\eta_{2}\right) \geq-A+\frac{1}{2} \ln \left(\frac{\bar{E}-\bar{d} N_{2}^{2}\left(\eta_{4}\right)}{\bar{d} M_{2}^{2}\left(\eta_{3}\right)}\right) .
\end{aligned}
$$

By (22) and (27),

$$
\begin{aligned}
& \int_{0}^{\omega}\left|u_{2}^{\prime}(t)\right| \mathrm{d} t \\
& =\lambda \int_{0}^{\omega}\left|-d(t)+\frac{E(t) e^{2 u_{1}\left(t-\sigma_{3}(t)\right)}}{M_{2}^{2}(t) e^{2 u_{2}\left(t-\sigma_{3}(t)\right)}+N_{2}^{2}(t) e^{2 u_{1}\left(t-\sigma_{3}(t)\right)}}\right| \mathrm{d} t \\
& \leq \int_{0}^{\omega}|d(t)| \mathrm{d} t \\
& \quad+\int_{0}^{\omega} \frac{E(t) e^{2 u_{1}\left(t-\sigma_{3}(t)\right)}}{M_{2}^{2}(t) e^{2 u_{2}\left(t-\sigma_{3}(t)\right)}+N_{2}^{2}(t) e^{2 u_{1}\left(t-\sigma_{3}(t)\right)}} \mathrm{d} t \\
& =(\widehat{d}+\bar{d}) \omega .
\end{aligned}
$$

Hence

$$
\begin{aligned}
& u_{2}(t) \leq u_{2}\left(\xi_{2}\right)+\int_{0}^{\omega}\left|u_{2}^{\prime}(t)\right| \mathrm{d} t t \in[0, \omega], \\
& \leq A+\frac{1}{2} \ln \left(\frac{\bar{E}-\bar{d} N_{2}^{2}\left(\xi_{4}\right)}{\bar{d} M_{2}^{2}\left(\xi_{3}\right)}\right)+(\widehat{d}+\bar{d}) \omega=: B_{1}, \\
& u_{2}(t) \geq u_{2}\left(\eta_{2}\right)-\int_{0}^{\omega}\left|u_{2}^{\prime}(t)\right| \mathrm{d} t \quad t \in[0, \omega] .
\end{aligned}
$$


So

$$
\left|u_{2}\right|_{0} \leq \max \left\{\left|B_{1}\right|,\left|B_{2}\right|\right\}=: B
$$

By (22),

$$
\begin{aligned}
& \left|u_{2}^{\prime}\right|_{0} \\
& \quad=\lambda\left|-d(t)+\frac{E(t) e^{2 u_{1}\left(t-\sigma_{3}(t)\right)}}{M_{2}^{2}(t) e^{2 u_{2}\left(t-\sigma_{3}(t)\right)}+N_{2}^{2}(t) e^{2 u_{1}\left(t-\sigma_{3}(t)\right)}}\right| \\
& \quad \leq|d|_{0}+\left|\frac{E}{N_{2}^{2}}\right|_{0}=: D_{2} .
\end{aligned}
$$

By (38), (43), (51), and (52),

$$
\|u\|=|u|_{\infty}+\left|u^{\prime}\right|_{\infty} \leq A+B+D_{1}+D_{2} .
$$

Consider the algebraic system

$$
\begin{gathered}
\bar{a}-\bar{B} e^{u_{1}}-\frac{1}{\omega} \int_{0}^{\omega} \frac{C(t) e^{u_{1}} e^{u_{2}}}{M_{1}^{2}(t) e^{2 u_{2}}+N_{1}^{2}(t) e^{2 u_{1}}} \mathrm{~d} t=0, \\
-\bar{d}+\frac{1}{\omega} \int_{0}^{\omega} \frac{E(t) e^{2 u_{1}}}{M_{2}^{2}(t) e^{2 u_{2}}+N_{2}^{2}(t) e^{2 u_{1}}} \mathrm{~d} t=0 .
\end{gathered}
$$

If $\left(u_{1}^{*}, u_{2}^{*}\right)^{T}$ is a solution of $(54)$, then there exist $\xi_{5}, \xi_{6} \in[0, \omega]$ such that

$$
\begin{gathered}
\bar{a}-\bar{B} e^{u_{1}^{*}}-\frac{\bar{C} e^{u_{1}^{*}} e^{u_{2}^{*}}}{M_{1}^{2}\left(\xi_{5}\right) e^{2 u_{2}^{*}}+N_{1}^{2}\left(\xi_{5}\right) e^{2 u_{1}^{*}}}=0, \\
-\bar{E}+\frac{\bar{E} e^{2 u_{1}^{*}}}{M_{2}^{2}\left(\xi_{6}\right) e^{2 u_{2}^{*}}+N_{2}^{2}\left(\xi_{6}\right) e^{2 u_{1}^{*}}}=0,
\end{gathered}
$$

which yields

$$
\begin{aligned}
& u_{1}^{*}=\ln \left(\frac{\bar{a}}{\bar{B}}-\frac{l \bar{C}}{\bar{B}\left[M_{1}^{2}\left(\xi_{5}\right) l^{2}+N_{1}^{2}\left(\xi_{5}\right)\right]}\right), \\
& u_{2}^{*}=u_{1}^{*}+\ln l
\end{aligned}
$$

where $l=\sqrt{\left(\bar{E}-\bar{d} N_{2}^{2}\left(\xi_{6}\right)\right) / M_{2}^{2}\left(\xi_{6}\right) \bar{d}}$. Further,

$$
\begin{aligned}
\left|u_{1}^{*}\right| & \leq \max _{t \in[0, \omega]}\left\{\left|\ln \frac{\bar{a}}{\bar{B}}\right|,\left|\ln \left\{\frac{1}{\bar{B}}\left[\bar{a}-\frac{1}{2} \bar{C}\left|\frac{1}{M_{1} N_{1}}\right|_{0}\right]\right\}\right|\right\} \\
& =: D_{3}, \\
\left|u_{2}^{*}\right| & \leq D_{3}+\max _{t \in[0, \omega]}\left\{| \operatorname { l n } \sqrt { \frac { \overline { E } - \overline { d } | N _ { 2 } ^ { 2 } | _ { 0 } } { \overline { d } | M _ { 2 } ^ { 2 } | _ { 0 } } | } , | \operatorname { l n } \left[\left|\frac{1}{M_{2}}\right|_{0} \sqrt{\left.\left.\frac{\bar{E}}{\bar{d}}\right] \mid\right\}}\right.\right. \\
& =: D_{4} .
\end{aligned}
$$

In addition, it is not difficult to see that the algebraic system

$$
\begin{gathered}
\bar{a}-\bar{B} e^{u_{1}}=0, \\
-\bar{d}+\frac{1}{\omega} \int_{0}^{\omega} \frac{E(t) e^{2 u_{1}}}{M_{2}^{2}(t) e^{2 u_{2}}+N_{2}^{2}(t) e^{2 u_{1}}} \mathrm{~d} t=0
\end{gathered}
$$

has a unique solution $\left(\widetilde{u}_{1}, \widetilde{u}_{2}\right)^{T}$. Let $F$ be a constant satisfying

$$
\left|\tilde{u}_{1}\right|+\left|\tilde{u}_{2}\right|<F
$$

Set $K=A+B+D_{1}+D_{2}+D_{3}+D_{4}+F$. Obviously, $K$ is independent of $\lambda$. Set

$$
\Omega=\left\{\left(u_{1}, u_{2}\right)^{T} \in X:\left\|\left(u_{1}(t), u_{2}(t)\right)^{T}\right\|<K\right\} .
$$

Clearly, the condition (i) in Lemma 3 holds. If $\left(u_{1}, u_{2}\right)^{T} \in$ $\partial \Omega \cap \operatorname{Ker} L=\partial \Omega \cap R^{2}$, then $\left(u_{1}(t), u_{2}(t)\right)^{T}$ is a constant vector in $R^{2}$ with $\left|u_{1}\right|+\left|u_{2}\right|=K$. Thus

$$
\begin{aligned}
& \text { QN }\left[\begin{array}{l}
u_{1} \\
u_{2}
\end{array}\right] \\
& \quad=\left[\begin{array}{c}
\bar{a}-\bar{B} e^{u_{1}}-\frac{1}{\omega} \int_{0}^{\omega} \frac{C(t) e^{u_{1}} e^{u_{2}}}{M_{1}^{2}(t) e^{2 u_{2}}+N_{1}^{2}(t) e^{2 u_{1}}} \mathrm{~d} t \\
-\bar{d}+\frac{1}{\omega} \int_{0}^{\omega} \frac{E(t) e^{2 u_{1}}}{M_{2}^{2}(t) e^{2 u_{2}}+N_{2}^{2}(t) e^{2 u_{1}}} \mathrm{~d} t
\end{array}\right] \\
& \quad \neq\left[\begin{array}{l}
0 \\
0
\end{array}\right] .
\end{aligned}
$$

So the condition (ii) in Lemma 3 holds.

Define the homology $\Psi: \operatorname{Dom} L \times[0,1] \rightarrow Z$ by

$$
\begin{aligned}
& \Psi\left(\left(u_{1}, u_{2}\right)^{T}, \mu\right) \\
& =\left(\begin{array}{c}
\bar{a}-\bar{B} e^{u_{1}} \\
-\bar{d}+\frac{1}{\omega} \int_{0}^{\omega} \frac{E(t) e^{2 u_{1}}}{M_{2}^{2}(t) e^{2 u_{2}}+N_{2}^{2}(t) e^{2 u_{1}}} \mathrm{~d} t
\end{array}\right) \\
& +\mu\left(-\frac{1}{\omega} \int_{0}^{\omega} \frac{C(t) e^{u_{1}} e^{u_{2}}}{M_{1}^{2}(t) e^{2 u_{2}}+N_{1}^{2}(t) e^{2 u_{1}}} \mathrm{~d} t\right) .
\end{aligned}
$$

Similar to the derivations of (57), for any $\left(u_{1}, u_{2}\right)^{T} \in \partial \Omega \cap$ Ker $L$ and $\mu \in(0,1), \Psi\left(\left(u_{1}, u_{2}\right)^{T}, \mu\right) \neq 0$. By (57) and (59), for any $\left(u_{1}, u_{2}\right)^{T} \in \partial \Omega \cap \operatorname{Ker} L$ and $\mu \in[0,1]$,

$$
\Psi\left(\left(u_{1}, u_{2}\right)^{T}, \mu\right) \neq 0
$$

Define the isomorphism $J=I: \operatorname{Im} Q \rightarrow \operatorname{Ker} L$ by $\left(u_{1}\right.$, $\left.u_{2}\right)^{T} \rightarrow\left(u_{1}, u_{2}\right)^{T}$. By homotopy invariance of topological degree, 
$\operatorname{deg}\{J Q N, \Omega \cap \operatorname{Ker} L, 0\}=\operatorname{deg}\{Q N, \Omega \cap \operatorname{Ker} L, 0\}$

$$
\begin{aligned}
& =\operatorname{deg}\left\{\Psi\left(\left(u_{1}, u_{2}\right)^{T}, 1\right), \Omega \cap \operatorname{Ker} L, 0\right\}=\operatorname{deg}\left\{\Psi\left(\left(u_{1}, u_{2}\right)^{T}, 0\right), \Omega \cap \operatorname{Ker} L, 0\right\}
\end{aligned}
$$

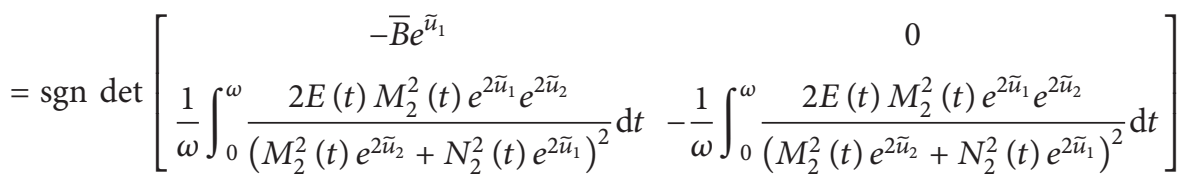

$$
\begin{aligned}
& =\operatorname{sgn}\left\{\frac{2 \bar{B} e^{3 \widetilde{u}_{1}} e^{2 \widetilde{u}_{2}}}{\omega} \int_{0}^{\omega} \frac{E(t) M_{2}^{2}(t)}{\left(M_{2}^{2}(t) e^{2 \widetilde{u}_{2}}+N_{2}^{2}(t) e^{2 \widetilde{u}_{1}}\right)^{2}} \mathrm{~d} t\right\} \\
& \neq 0 \text {. }
\end{aligned}
$$

So, the condition (iii) of Lemma 3 holds. By Lemma 3, there exists one $\omega$-periodic solution of system (13). The proof of Theorem 4 is complete.

Remark 5. From the proof of Theorem 4, we can find that Theorem 4 is also true if $\theta_{1 k}=\theta_{2 k}=0, k \in N^{+}$. Therefore, we can obtain the following result.

Corollary 6. Suppose that $\left(H_{1}\right)$ and the following conditions hold.

$\left(\mathrm{H}_{6}\right)$ For $t \in[0, \infty), \sigma_{1}^{\prime}(t)<1, \sigma_{2}^{\prime}(t)<1$, let $t=\phi_{1}(p)$ be the inverse function of $p=t-\sigma_{1}(t)$ and $W(t)>V^{\prime}(t)$, where $W(t)=b\left(\phi_{1}\left(t-\sigma_{2}(t)\right)\right)\left(1-\sigma_{2}^{\prime}(t)\right) /\left(1-\sigma_{1}^{\prime}\left(\phi_{1}(t-\right.\right.$ $\left.\left.\left.\sigma_{2}(t)\right)\right)\right)$ and $V(t)=\rho(t) /\left(1-\sigma_{2}^{\prime}(t)\right)$.

$\left(\mathrm{H}_{7}\right)$ Consider $|\rho|_{0} e^{A_{3}}<1$, where $A_{3}=\ln \left[2 \bar{a} \max _{t \in[0, \omega]}\{(1-\right.$ $\left.\left.\left.\sigma_{2}^{\prime}(t)\right) /\left(W(t)-V^{\prime}(t)\right)\right\}\right]+2 \bar{a}|V|_{0} \max _{t \in[0, \omega]}\{1 /(W(t)-$ $\left.\left.V^{\prime}(t)\right)\right\}+(\widehat{a}+\bar{a}) \omega$.

$\left(\mathrm{H}_{8}\right)$ Consider $2 \bar{a}>\bar{c}|1 / m|_{0}$ and $\bar{e}>\bar{d}$.

Then the following neutral ratio-dependent predator-prey system with Holling type III functional response

$$
\begin{aligned}
& x^{\prime}(t) \\
& =x(t)\left[a(t)-b(t) x\left(t-\sigma_{1}(t)\right)-\rho(t) x^{\prime}\left(t-\sigma_{2}(t)\right)\right] \\
& \quad-\frac{c(t) x^{2}(t)}{m^{2}(t) y^{2}(t)+x^{2}(t)} y(t), \\
& y^{\prime}(t) \\
& =y(t)\left[-d(t)+\frac{e(t) x^{2}\left(t-\sigma_{3}(t)\right)}{m^{2}(t) y^{2}\left(t-\sigma_{3}(t)\right)+x^{2}\left(t-\sigma_{3}(t)\right)}\right]
\end{aligned}
$$

has at least one positive $\omega$-periodic solution.

Remark 7. If $\sigma_{1}(t), \sigma_{2}(t), \rho(t), m(t)$ are all constants, then Corollary 6 matches Theorem 2.1 in [13]. Therefore, our main results extend Theorem 2.1 in [13].

\section{Conclusion}

In this paper, we construct a ratio-dependent predatorprey model with Holling type III functional response based on system of neutral impulsive differential equations. By constructing suitable homology and applying some analysis techniques and homotopy invariance of topological degree, we give the estimate on a priori bounds of periodic solutions for the corresponding system. Then the existence of positive periodic solutions for this model is obtained by applying coincidence degree theory.

Our main results demonstrate that under the suitable periodic impulse perturbations, the neutral impulsive system preserves the periodicity of the corresponding neutral system without impulse. In addition, our results can be applied to the corresponding neutral ratio-dependent predator-prey model without impulsive effect. Further, our results extend some results in the literature.

\section{Conflict of Interests}

The authors declare that there is no conflict of interests regarding the publication of this paper.

\section{Acknowledgments}

This work was supported by the Program for the Top Young Academic Leaders of Higher Learning Institutions of Shanxi (no. 20120304), Research Project Supported by Shanxi Scholarship Council of China (no. 2013-019), and Natural Science Foundation of Shanxi Province (no. 2014011005-1).

\section{References}

[1] V. Lakshmikantham, D. D. Bainov, and P. S. Simeonov, Theory of Impulsive Differential Equations, World Scientific, Singapore, 1989.

[2] Z. Liu and S. Zhong, "An impulsive periodic predator-prey system with Holling type III functional response and diffusion," Applied Mathematical Modelling, vol. 36, no. 12, pp. 5976-5990, 2012.

[3] X. Fan, F. Jiang, and H. Zhang, "Dynamics of multi-species competition-predator system with impulsive perturbations and 
Holling type III functional responses," Nonlinear Analysis: Theory, Methods \& Applications, vol. 74, no. 10, pp. 3363-3378, 2011.

[4] M. U. Akhmet, M. Beklioglu, T. Ergenc, and V. I. Tkachenko, "An impulsive ratio-dependent predator-prey system with diffusion," Nonlinear Analysis: Real World Applications, vol. 7, no. 5, pp. 1255-1267, 2006.

[5] X. Wang, W. Wang, and X. Lin, "Dynamics of a periodic Watt-type predator-prey system with impulsive effect," Chaos, Solitons \& Fractals, vol. 39, no. 3, pp. 1270-1282, 2009.

[6] S. H. Saker and J. O. Alzabut, "Existence of periodic solutions, global attractivity and oscillation of impulsive delay population model," Nonlinear Analysis: Real World Applications, vol. 8, no. 4, pp. 1029-1039, 2007.

[7] S. Ahmad and I. M. Stamova, "Asymptotic stability of an $\mathrm{N}$ dimensional impulsive competitive system," Nonlinear Analysis: Real World Applications, vol. 8, no. 2, pp. 654-663, 2007.

[8] J. Hou, Z. Teng, and S. Gao, "Permanence and global stability for nonautonomous $N$-species Lotka-Valterra competitive system with impulses," Nonlinear Analysis: Real World Applications, vol. 11, no. 3, pp. 1882-1896, 2010.

[9] R. Shi, X. Jiang, and L. Chen, "A predator-prey model with disease in the prey and two impulses for integrated pest management," Applied Mathematical Modelling, vol. 33, no. 5, pp. 2248-2256, 2009.

[10] H. Su, B. Dai, Y. Chen, and K. Li, "Dynamic complexities of a predator-prey model with generalized Holling type III functional response and impulsive effects," Computers \& Mathematics with Applications, vol. 56, no. 7, pp. 1715-1725, 2008.

[11] H. Baek, "Species extinction and permanence of an impulsively controlled two-prey one-predator system with seasonal effects," Biosystems, vol. 98, no. 1, pp. 7-18, 2009.

[12] Z. Du and Z. Feng, "Periodic solutions of a neutral impulsive predator-prey model with Beddington-DeAngelis functional response with delays," Journal of Computational and Applied Mathematics, vol. 258, pp. 87-98, 2014.

[13] G. Liu and J. Yan, "Positive periodic solutions for neutral delay ratio-dependent predator-prey model with Holling type III functional response," Applied Mathematics and Computation, vol. 218, no. 8, pp. 4341-4348, 2011.

[14] R. E. Gaines and J. L. Mawhin, Coincidence Degree and Nonlinear Differential Equations, Lecture Notes in Mathematics, Vol. 568, Springer, Berlin, Germany, 1977. 


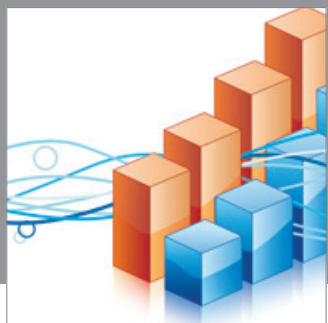

Advances in

Operations Research

mansans

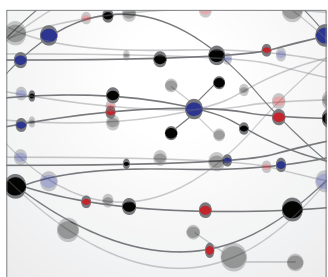

The Scientific World Journal
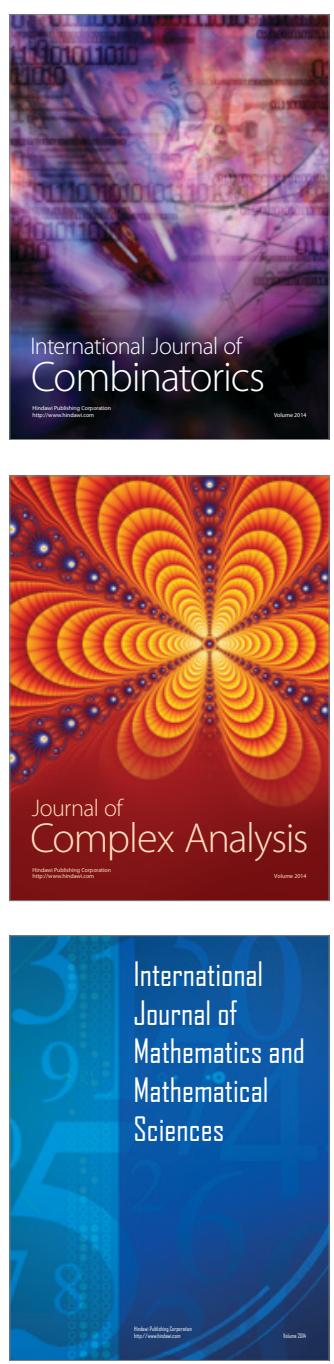
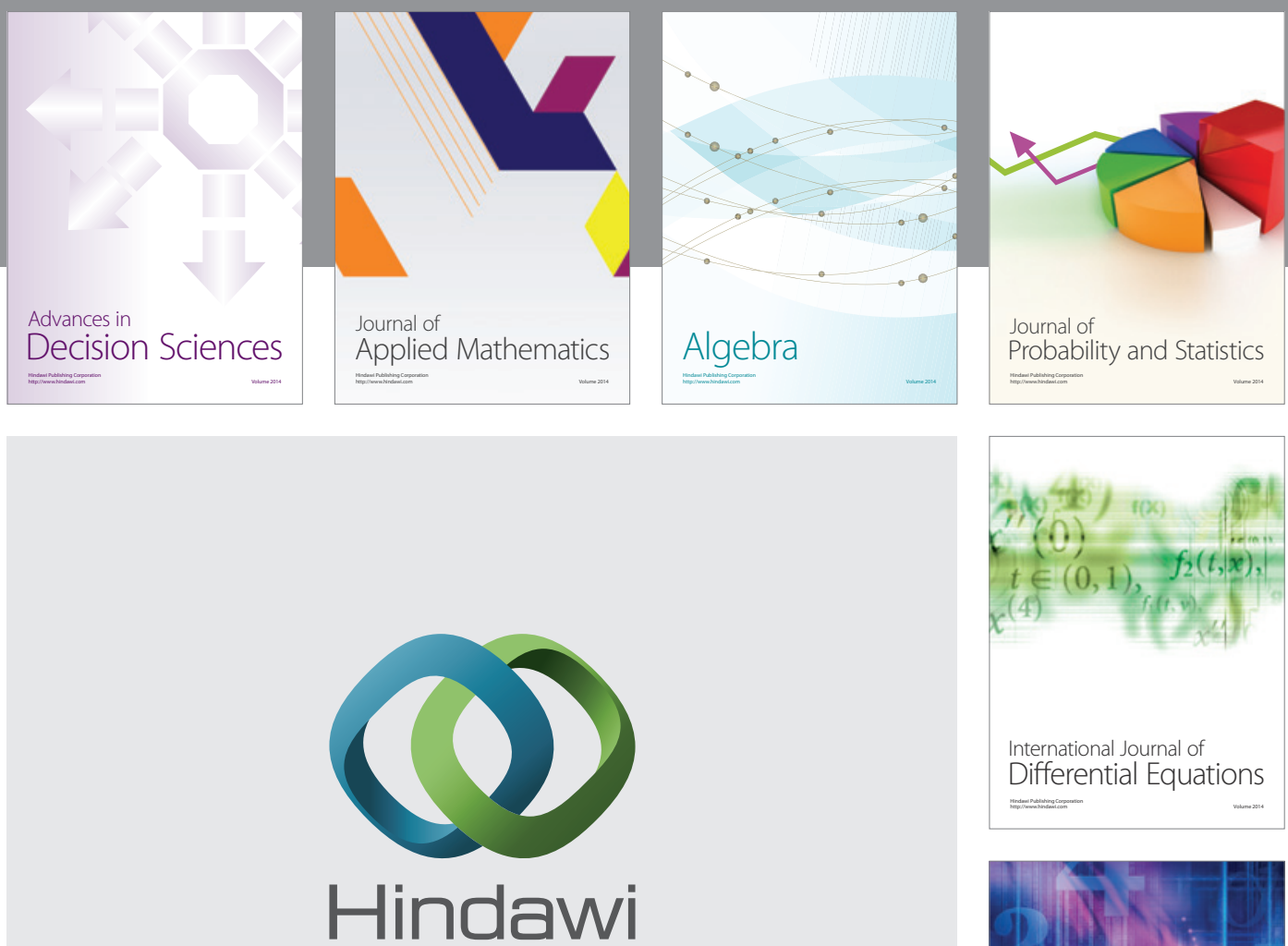

Submit your manuscripts at http://www.hindawi.com
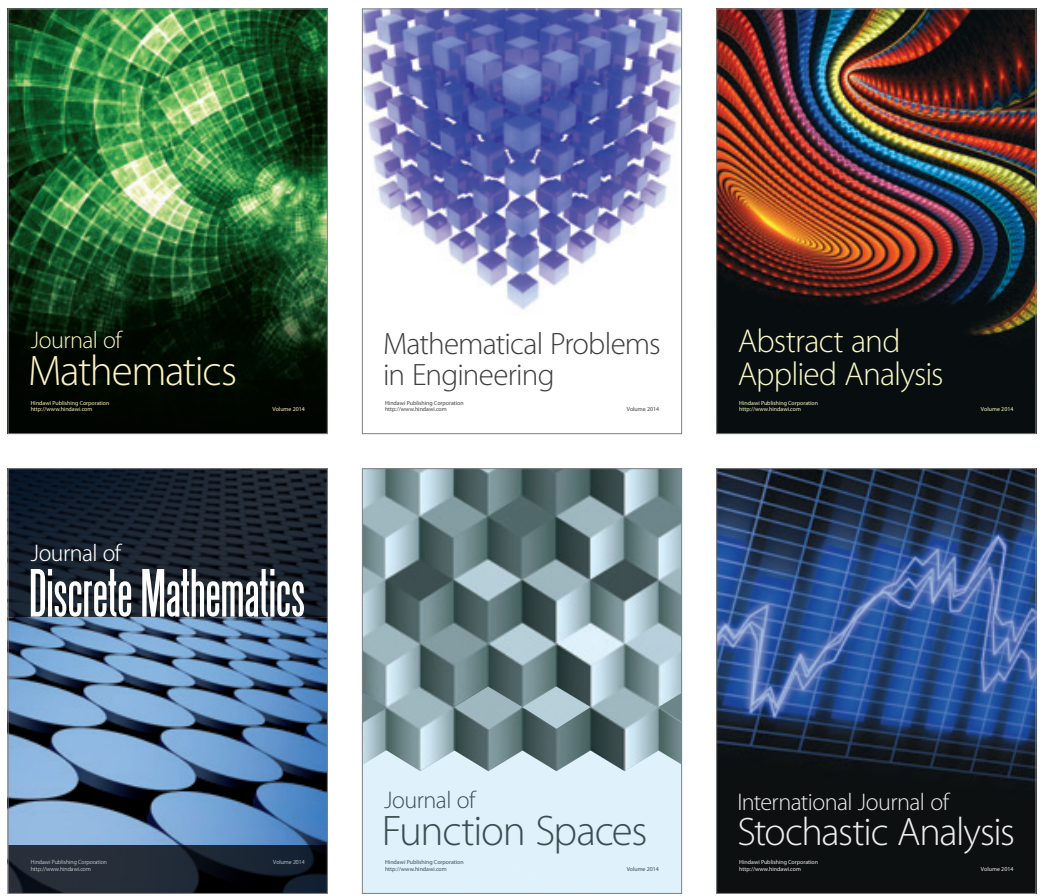

Journal of

Function Spaces

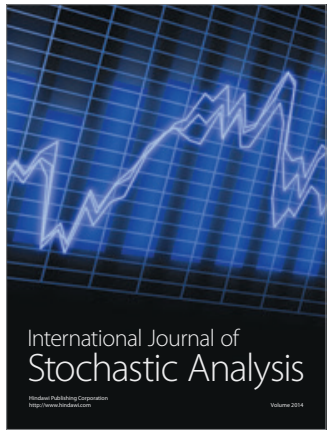

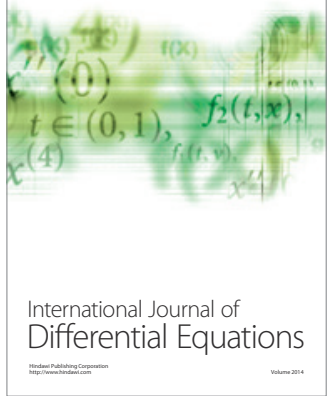
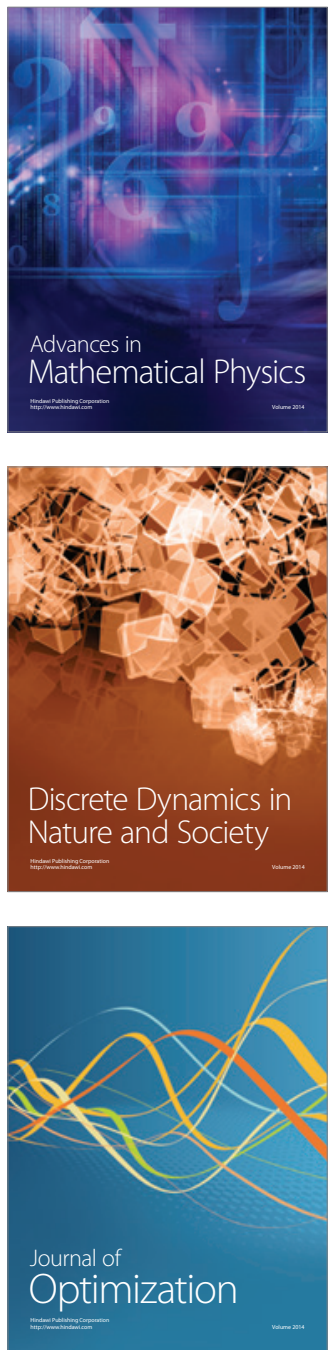